\title{
West, Harry G. - Ethnographic Sorcery
}

Chicago, The University of Chicago Press, 2007, 128 p.

\section{Camilla Strandsbjerg}

\section{CpenEdition}

\section{Journals}

Édition électronique

URL : https://journals.openedition.org/etudesafricaines/9592

DOI : $10.4000 /$ etudesafricaines.9592

ISSN : $1777-5353$

Éditeur

Éditions de l'EHESS

Édition imprimée

Date de publication : 7 avril 2008

Pagination : 378-381

ISBN : 978-2-7132-2141-5

ISSN : 0008-0055

\section{Référence électronique}

Camilla Strandsbjerg, « West, Harry G. - Ethnographic Sorcery », Cahiers d'études africaines [En ligne], 189-190 | 2008, mis en ligne le 16 avril 2008, consulté le 10 mai 2021. URL : http://

journals.openedition.org/etudesafricaines/9592 ; DOI : https://doi.org/10.4000/etudesafricaines.9592

Ce document a été généré automatiquement le 10 mai 2021.

(c) Cahiers d'Études africaines 


\title{
West, Harry G. - Ethnographic Sorcery
}

\author{
Chicago, The University of Chicago Press, 2007, 128 p.
}

\section{Camilla Strandsbjerg}

1 Depuis la fameuse étude menée par E. E. Evans-Pritchard à la fin des années 1920 où l'ethnologue britannique s'efforce de montrer la rationalité des croyances magiques des Azande $^{1}$, les phénomènes dits de sorcellerie n'ont cessé d'intriguer les anthropologues. Au cours du $\mathrm{xx}^{\mathrm{e}}$ siècle, le changement des paradigmes théoriques a modifié à plusieurs reprises les analyses de la sorcellerie, tandis que les questions épistémologiques relatives à ce sujet de recherche ont moins retenu l'attention. Tout récemment on a assisté à une multiplication des publications d'ouvrages anthropologiques consacrés à « l'occulte » et à la sorcellerie qui pourrait même donner l'impression d'une certaine sorcellerie des anthropologues ${ }^{2}$ ! C'est cependant dans une perspective inverse - celle de la sorcellerie ethnographique ou plus précisément de l'ethnographie sorcière!- que Harry G. West exploite les implications épistémologiques des phénomènes de sorcellerie de façon assez originale.

2 Dans ce petit volume (132 pages), prévu initialement comme un chapitre de son ouvrage principal Kupilikula: Governance and the Invisible Realm in Mozambique (2005) ${ }^{3}$, l'auteur a opté pour une forme d'anthropologie narrative, voire fictive, combinant le récit de son expérience personnelle - comme il l'a vécue lors de ses terrains au nord du Mozambique depuis $1994-$ avec des notions théoriques et analytiques de l'anthropologie ainsi qu'une bibliographie et un appareil de notes relativement riches. Par cette double démarche, il se distingue notablement de précédentes tentatives de réviser le rapport entre l'ethnographe et cet objet « magique » de la sorcellerie, comme par exemple celle de Paul Stoller et Cheryl Olkes In Sorcery's Shadow: A Memoir of Apprenticeship among the Songhay of Niger (1989) ${ }^{4}$. Toutefois, les deux ouvrages s'inscrivent dans une approche assez similaire comparant le savoir ethnographique à celui du sorcier.

3 À travers les quinze brefs chapitres de l'ouvrage, l'auteur s'interroge sur la nature des phénomènes de sorcellerie en soulevant de nombreuses questions relatives à l'enquête de terrain et à la méthodologie de l'anthropologie de manière plus générale. Le mode narratif (storytelling) du livre permet de se déplacer continuellement entre le regard du 
chercheur, de son interprète et de leurs interlocuteurs, ce qui est particulièrement intéressant lors de la "restitution » des conversations avec les "contre-sorciers "/ guérisseurs. Ce trait stylistique constitue certainement tout l'intérêt et la force du livre, mais par moments aussi sa faiblesse. Autrement dit, la manière dont l'auteur déplace la perspective entre ces différents plans du récit mais aussi de l'analyse, rend le livre très riche et la lecture agréablement vivante, mais par moments aussi les propos de l'auteur moins clairs.

Dans le premier chapitre, intitulé Misunderstandings (Malentendus) le lecteur est placé de façon très convaincante au cœur de la thématique du livre: le rapport entre le savoir académique et le "savoir local» (ou plutôt selon le terme anglais: local understandings). Lors d'une séance de restitution de sa recherche au sein d'un groupe de chercheurs et de fonctionnaires mozambicains, West rend compte de son analyse des " hommes-lions » apparus à plusieurs reprises dans cette région du Mozambique ${ }^{5}$. Face à la perspective turnerienne du chercheur étranger insistant sur le caractère symbolique du phénomène, l'audience proteste en signalant qu'il a «mal compris » puisque ces lions ne sont pas des « symboles », mais qu'ils sont bien « réels» (p. 5).

Cette entrée dans le sujet sera alors la base d'une série d'interrogations sur le caractère de la sorcellerie. À cet égard, le chapitre sur les possibles appréhensions en termes "scientifiques » de la sorcellerie (chapitre 3) et le rappel de l'éternelle question de la " croyance " personnelle du chercheur à la sorcellerie (chapitre 4) sont moins concis que ceux consacrés par la suite à la discussion du caractère métaphorique du discours de la sorcellerie - une question que West traite à plusieurs reprises de façon de plus en plus nuancée tout au long de l'ouvrage en s'appuyant sur les travaux de nombreux chercheurs dont Luise White (2000), Comaroff (1985), Jackson (1989)6.

6 Vient ensuite le récit de l'emprise de la "sorcellerie " sur l'auteur même et plus précisément du vécu d'une maladie, interprétée ultérieurement par son interprète en termes d'attaque sorcellaire, passant par l'expérience de guérison chez plusieurs "contre-sorciers"/guérisseurs rencontrés auparavant lors de l'enquête de terrain. L'auteur poursuit alors son approche particulière du personnage du «sorcier » (et du "contre-sorcier»), tout en abordant la «sorcellerie», comme dans ses écrits précédents, en tant que « discours de et sur la sorcellerie ».

7 C'est précisément cette perspective langagière, discursive, sur «la sorcellerie » qui ouvre la voie à une lecture en termes d'« ethnographie sorcière ». C'est-à-dire, adopter en fin de compte une position consistant à dire que les activités du sorcier et de l'ethnographe se ressemblent dans la mesure où les deux sont préoccupés par l'interprétation, voire par l'organisation (et la réorganisation) de la réalité sociale des autres. En possédant et en activant un savoir particulier, ils adhérent, selon West, à un espace physique et métaphysique en dehors de celui des gens ordinaires (p. 80).

C'est donc en adoptant en quelque sorte le point de vue des « sorciers » et de « contresorciers "/guérisseurs fréquentés lors de son enquête, qui voient en lui un « collègue ", voire un sorcier (selon West), que l'auteur arrive à son point principal assez intéressant, mais aussi à certains égards problématique. Il est vrai qu'à la fois le chercheur et le (contre-)sorcier considèrent la sorcellerie "comme une chose fabriquée » (p. 80) et que les activités de ces deux figures consistent d'un certain point de vue à « faire et à défaire la réalité sociale des autres » (p. 80) en cherchant à gagner "une ascendance interprétative du monde» (p. 80)7. En «articulant leurs visions du monde» (pp.55-60) ils rendent explicites les pratiques sociales des autres (p. 57). 
Cependant la manière dont West prolonge cette perspective en citant les cas où luimême a agi en tant que "guérisseur " traitant effectivement sur le terrain les divers maux de la population du mieux qu'il pouvait, semble pousser l'analogie un peu trop loin. En outre, on pourrait se demander si ce rapprochement entre les personnages du chercheur et du sorcier n'en dit pas plus sur la relation entre les enquêtés et l'enquêteur - notamment à l'égard de la relation de pouvoir qui se construit à travers les échanges entre l'ethnographe et ses interlocuteurs (voir en particulier p. 85), que sur la sorcellerie comme objet anthropologique? En particulier il convient de se demander si la sorcellerie se résume à des pratiques langagières, à un langage que l'étranger, l'ethnographe en l'occurrence, peut apprendre pour alors comprendre la société (p. 11).

9 Dans tous les cas, au cours de la lecture, on se demande si l'auteur serait arrivé à faire cette analogie entre l'ethnographe et le sorcier (ou le contre-sorcier) s'il s'était penché sur une dimension de la sorcellerie autre que celle du discours, de la sorcellerie comme langage? Force est de constater que les études sur «la sorcellerie» continuent à proposer des angles d'analyse fort divers, comme en témoigne l'ouvrage dirigé par Gerrie ter Haar abordant la sorcellerie sous l'angle des droits de l'Homme et des tragédies humaines impliquées dans des accusations et des meurtres de sorciers supposés par leur entourage ${ }^{8}$. Si les deux livres représentent deux approches totalement opposées, ce qui rend la comparaison peu constructive, leur différence n'en témoigne pas moins de la «largeur » du phénomène dit de sorcellerie. Un objet qu'il convient probablement de nuancer davantage pour comprendre les enjeux en question et surtout pour proposer une démarche analytique constructive d'un point de vue scientifique.

\section{NOTES}

1. E. E. EVAns-PRItchaRd, Sorcellerie, oracles et magie chez les Azandé, Paris, Gallimard, 1972.

2. Voir aussi Terence RANGER, « Scotland Yard in the Bush : Medicine Murders, Child Witches and the Construction of the Occult : A Literature Review », Africa, 77, 2007, pp. 272-283.

3. Harry G. WEST, Kupilikula : Governance and the Invisible Realm in Mozambique, Chicago, Chicago University Press, 2005.

4. Paul STOLLER \& Cheryl OLKES, In Sorcery's Shadow: A Memoir of Apprenticeship among the Songhay of Niger, Chicago, Chicago University Press, 1989.

5. Voir aussi la contribution de Paolo ISRAËL dans ce numéro.

6. Luise WHITE, Speaking with the Vampires : Rumor and History in Colonial Africa, Berkeley, University of California Press, 2000 ; Jean CoMAROFF, Body of Power, Spirit of Resistance: The Culture and History of a South African People, Chicago, Chicago University Press, 1985 ; Michael JACKSON, Paths toward a Clearing: Radical Empiricism and Ethnographic Inquiry, Bloomington-Indianapolis, Indiana University Press, 1989. 
7. Rappelons que cette perspective constructiviste imprègne l'ensemble du travail de West. Pour une présentation de la façon dont le discours de la sorcellerie peut « inverser » les rapports de pouvoir, voir aussi H. G. WEST, « Creative Destruction and Sorcery of Construction : Power, Hope and Suspicion in Post-War Mozambique ", Cahiers d'Études africaines, XXXVII (3), 147, 1997, pp. 13-31.

8. Gerrie TER HAAR (ed.), Imagining Evil. Witchcraft Beliefs and Accusations in Contemporary Africa, Trenton, Africa World Press, 2007 (voir le compte rendu sur cet ouvrage dans ce numéro). 